INFORMATION PROCESSING MODELS: BENEFITS AND LIMITATIONS

\author{
Brendan Wallace, Alastair Ross, John Davies \\ Brendan Wallace, Alastair Ross, John Davies \\ University of Strathclyde \\ 141 St James Road \\ Glasgow G4 OLT
}

\begin{abstract}
This paper looks at the three main information processing models from the point of view of researchers in confidential human factors databases. It explores conceptual problems with two of these information processing models, and goes on to explore possible advantages of adopting a 'connectionist' paradigm. Links between connectionism and 'situated cognition' are demonstrated. Practical work carried out using a connectionist/situated cognition model is described, and the way in which the 'situatedness' of discourse can influence the kind of data that can be collected is discussed. Finally it is argued that more emphasis should be placed in ergonomics on sociation, situatedness and embodiment, and that this might help to deal with problems faced in creation and interrogating databases: especially as regards the creation of coherent and reliable 'coding taxonomies'.
\end{abstract}

\title{
Introduction
}

The information processing approach to human cognition remains very popular in the field of ergonomics. However, in recent years, an alternative approach has arisen: 'situated cognition'. Situated cognition stresses the extent to which human beings related dynamically with their environment (Hollnagel, in press). However, there are few generalised discussions of the advantages of both approaches in a pragmatic human factors context.

The purpose of this paper is to show that there are generally agreed to be three main information-processing approaches, and that at least one seems to be compatible with situated cognition. We will then go on to discuss how useful the situated cognition approach has been in our own work. Finally there will be a brief discussion of the implications for ergonomics.

\section{Information Processing}

Our work at the University of Strathclyde has been with qualitative research in safety management and human factors. There are three major projects we have been involved with which are of importance here. Firstly there is CIRAS. CIRAS is the Confidential Incident Reporting and Analysis System, the national confidential reporting system for the UK Railways. The University of Strathclyde set up the system and established it as a national database (Davies, et al, in press). We have also run a number of focus group studies of train drivers within the railway industry (Wallace et al, 2002). We have also just begun to run CARA (Confidential Accident Reporting and Analysis), the nation-wide confidential reporting system for the railway system of the Republic of Ireland.

It was because we come from a psychology background that we looked to information processing theories for a theory of 'human error'. However (as is not noted frequently enough in discussions on this subject) there are not one but three main information processing theories.

The best known of these is the Atkinson and Shiffrin model (1968). This posits memory as having two main components. Firstly there is a short-term memory (STM) store, which has a limited amount of storage space. Data is inputted into this store, before being passed onto the long-term memory (LTM) store that has an infinite capacity. Because of this distinction, this is normally known as the two stage, or 'modal' memory model. 
However, there are a number of problems with the concept of two distinct systems of memory. Here we must remember a number of correlates of Atkinson and Shiffrin's theories. For example, by definition, data must pass through the short-term store, before passing into the long-term store. But as Shallice and Warrington (1970) have showed, it is perfectly possible for some brain-damaged patients to have perfectly normal long-term memory but no short-term memory. This is impossible in the Atkinson and Shiffrin theory.

Of course, there are many experiments that purport to demonstrate that there are indeed two memory stores. However, they can usually be interpreted in other ways, ways that fit more closely with 'ecological' studies of how human beings actually function in their 'natural environments'. For example, Reisberg, Rappaport, and O'Shaughnessy (1984) demonstrated that it is easy, by using their fingers as aides memoires, to improve the capacity of the 'short term memory store' immensely. As Glenberg (1997) comments (in a very important paper to which we will return) 'This evidence might be interpreted as evidence for a new "finger-control" module, but it seems more sensible to view it as a newly-learned skill' (italics added). This is a very important point: memory behaviour is better seen as being a skill or activity that can improve through practice. And if this is the case, then talking about the 'absolute capacity' of short-term memory store, as though it can be 'filled up' with digital data seems to be meaningless. As Glenberg writes: '(in recent years) much of the evidential basis for a separate short- term (memory) store has been eroded' (Glenberg, 1998). Given that this is the case, then simply renaming STM 'working memory' will not solve the problem, although 'working memory' does solve, ironically enough, a far deeper problem with the whole concept: the idea of memory as a store (Baddeley, 1986).

It was as a result of such problems that an alternative Craik and Lockhart (1972) model of memory was proposed. It is generally agreed that it at least blurs, and possibly eliminates the STM/LTM distinction (Hayes, 2000), positing instead a single memory store. In this model, things which are processed more deeply are better remembered than those which are not. It therefore avoids the STM/LTM problem above.

But there is a deeper problem with both these theories, and one which cuts to the root of the matter: is memory a store at all? The problem here is conceptual and empirical. To take the conceptual problem first: The key point to remember is that in order to 'recognise' something, both the 'information processing' models described propose that the operator must retrieve a stored memory from a memory store, and then match it with the information in front of him or her. If it matches, then the object is 'remembered'.

However, there is a fundamental problem with this approach, which demonstrates its reliance on the now outmoded 'cognitivist' model of cognition. Cognitivism was discarded because it fell prey to the 'homunculus' fallacy: it posited an internal 'person' who provided the motor for cognition. However, this then led to infinite regress (who controlled the homunculi? Obviously, other homunculi). (Davies et al, in press. See also Dreyfus 1992 and Barba, 1999).

The concept of a memory store seems to fail for the same reason. If the operator sees their car, for example, how do they know where it should be looked for in the memory store, in order to 'remember' it? S/he would have to remember that it was his/her car (and, in fact that it was a car at all) before the search through the memory store for 'my car' could begin. The alternative is that the entire memory store would have to be searched through for every act of recognition.

Even if the memory was retrieved, how would the operator know it was the 'correct' memory? The answer, never stated but implicit, is that a homunculus would compare the internal 'symbol' in the memory store with the symbol of the external object, because the homunculus would remember what a 'car' looked like and be able to compare it with the external symbol. In other words, they object would have to be recognised (and therefore remembered) before it can be compared in the store: what the model is attempting to explain (Wilcox and Katz, 1981).

Moreover the concept of memory 'stores' seems to be empirically false as well. If memory is a store, it is simply a passive receptacle of events. But Bekerian and Baddeley (1980) conducted a classic study which studied how new radio frequencies were remembered. 
Despite bombardment by the media, people still did not retain the information, which suggests that the theory that communication is simply a matter of repeating information often enough until it is 'put into' the store, is false.

\section{The Third Information Processing Model}

There is, however, a third information processing model. This is the paralleldistributed processing (PDP) or 'connectionist' model. There are two main differences between connectionist and the other two main information processing models. Firstly, in connectionist models, memories are not 'stored' but instead are distributed throughout the system (Bechtel and Abrahamson, 2002). Secondly, connectionist models posit one, not two, memory 'systems' (Sougné, 2000).

We have discussed connectionism extensively elsewhere (see Davies et al, in press). However, the key point to realise is that connectionist models are conceptually plausible in a way that the other two models of information processing are not. Moreover, as far as a connectionist system's processing is concerned, 'there is no difference between reconstructing a previous state, and constructing a completely new state (confabulating)' (Bechtel and Abrahamsen, 2002: 50). In other words, in the connectionist view memory is a dynamic act of reconstruction (Clancey, 1997). A number of experiments have demonstrated these points (for example, Loftus and Loftus, (1975), see also Zadny and Gerard (1974))

To return to Glenberg's paper, he argues, on the basis of the embodied cognition theory of Lakoff and Johnson (1999), that: 'memory evolved in service of perception and action...and that memory is embodied to facilitate interaction with the environment' (Glenberg, 1998). Memory is a tool which facilitate the organism in dealing with the environment, and we would argue that this is a good model for all behaviour, linguistic or otherwise.

As we have seen, connectionist models avoid the most obvious problems resulting from the other two 'information processing' approaches. However, situated cognition goes further by stressing the situated aspect of language and behaviour: the extent to which these are specific responses to specific situations. The main aspects of this model we have found useful in our own research is that of the situatedness of discourse.

\section{Accident Investigation}

It tends to be generally accepted within the field of safety management that discourse about past events (what one might term memory discourse) is veridical, that is, that discourse is an attempt to communicate the 'truth' from one subject to another. But this misses an important point about communication; that is, as stated above, the extent to which memory is an active, situated response to a situation.

Despite its expression in the language of situated cognition, this insight would strike most non-psychologists as being common sense, especially in a situation such as an accident investigation, where people still attempt to deny that they were 'to blame' when investigations try to reveal 'what caused the accident'. For example, in a study of a major train operator (Wright, Ross and Davies 2001), discussion groups were held with drivers who had passed a signal at danger and those who had not, in order to establish risk factors for Signals Passed at Danger (SPADs). Drivers with no SPADs on their driving record stated that they had been caused more by dispositional factors (i.e. things to do with the other drivers, such as inability to concentrate) than the drivers who had previous SPADs. By contrast, this latter group remembered system and environmental conditions (e.g. training, railhead conditions) for their own incidents, thus implying that SPADs were "caused" by external factors beyond their control. Thus memory discourse of SPADs seemed to be presented in a functional manner and showed a self-serving bias, dependent on drivers' own 
personal histories. This is not to suggest that drivers were 'lying'. Instead it must be remembered that all discourse exists in a specific situation, and that it was created to cope with the needs of that situation. Consciously or not, any subject will tend to remember things (to be precise, produce memory type discourse) which is situation specific and which facilitates their own goals (this of course applies to management as much as to workers at the man-machine interface.) This research suggests is that approaches to safety which suggest that the purpose of discourse in situations such as these as primarily veridical are flawed.

One way of dealing with this problem is to treat discourse (instead of being veridical or otherwise) as being 'stable' or 'unstable'. That is, to acknowledge that there are certain kinds of discourse that will be produced in any normal situation (for example, the subject's name): this is 'stable' data. Other kinds of discourse will only be produced in specific situations (for example, in this respect, the answer to the question 'why did you have a SPAD?' will be very different depending on whether the subject is in a courtroom during an inquiry, or in the pub with co-workers): this is 'unstable'. Therefore a process of 'triangulation' can be used, in which data are obtained from subjects in different situations. This does not of course have to be done for every fragment of data or with every subject. But even a small scale study might indicate the relationship between stable and unstable data, and help to give an insight regarding the extent to which the data obtained in research is stable or not (for details as to how this might be done, see Davies, 1997).

\section{Safety Culture}

Another way of using situated discourse is to see whether it can give help in terms of defining safety culture. Despite common usage, there is no agreed definition of 'safety culture'. Indeed Cummings and Worley (1997: 479) state that 'Despite the increased attention and research devoted to corporate culture, there is still some confusion about what the term culture really means when applied to organisations' (emphasis as original).

What we would suggest here is that safety culture could be interpreted as the social complex of intersubjective meanings of an organisation expressed through language. In other words, the reconstructions of 'memory', or rather the linguistic behaviours we call 'memory behaviours' are an indication of the safety culture itself. That is, if a reporter chooses to remember an event as being managerial in origin, rather than being 'his own fault', then this can be studied in its own terms (it must be remembered that CIRAS and CARA are confidential systems and so no 'check' on the veridical nature of the memories can be performed). Therefore, we can study texts of memory events as texts to be classified and coded.

We have described elsewhere our methodology for transcribing and coding texts (Davies et al, in press) and performing a consensus or reliability trial. However, the key point is that codings must be reliable (i.e. socially agreed upon). The matrix produced in figure 1 in order to code the transcriptions is a general ethnographic matrix: detailed codes are project specific. This, therefore, is the JCP (Job, Communications, Procedures) 'coding matrix' which produces data.

\begin{tabular}{|l|l|l|l|}
\hline & Job/Task & Communications & Procedures \\
\hline Managerial & Codes & Codes & Codes \\
\hline Supervisory & Codes & Codes & Codes \\
\hline Frontline & Codes & Codes & Codes \\
\hline
\end{tabular}

Figure 1. The JCP coding matrix currently used in CARA

Once the discourse has been coded and entered into a database, then the data can be analysed. Analysis in this way is therefore an ethnographic (and hence, interpretative) classification taxonomy of discourse. In this discourse reporters write in and recreate events and situations which happened to them, or that they saw or experienced. These may be 'true' 
or 'false' but of course because of the confidential nature of the database, we can never know this.

\section{Reliability and Discussion}

We have found situated cognition (or rather, situated behaviour and discourse) a useful model for our work (not least in terms of the 'embodiment' of cognition as stressed by Lakoff and Johnson (1999) a topic which lies outside the scope of this paper. See however, Wallace et al 2002). But the key point in terms of discourse is that of consensus or reliability. It is taken for granted within most fields of the social sciences that in situations where behaviour, either in terms of physical behaviour or linguistic behaviour, has to be 'judged' or 'coded', that coders have to agree with each other. In other words, two coders coding the same text would code it the same way, all other things being equal. However we found consensus impossible to achieve when attempting to use 'information processing' concepts to create a matrix of 'human error'. Situated concepts on the other hand, proved a far better model as the situation they reporters were in (the workplace) could more easily be verified from the reports. It should be noted that the matrix in figure 1 is an organisational matrix which does not posit discrete 'internal states' which 'cause' 'human error'.

Another implication of this research is that discourse and behaviour are situated. One way of dealing with this has already been suggested: triangulation. However if this is not possible, the situatedness of behaviour should at least be controlled for. That is, if a database is being built up based on discourse (or from questionnaires, which are simply tabulations of discourse), the discourse producing situations should be as similar as possible.

This must be stressed, as, during research still ongoing, we have discovered that databases concerning the 'causes' of car accidents are frequently compared to each other, regardless of how the data was collated. For example, some police forces specifically ask drivers involved in accidents questions regarding whether certain 'risk factors' were present or absent (for example, if the driver was using a mobile phone at the time of the accident). Others do not, and only note this data if the driver volunteers the information. But it is clear that differences between these databases do not reflect 'real' differences in accident causes, but demand characteristics of the situations itself. How frequently will drivers volunteer information that may land them in court? However, data from these two sources is frequently compared as though one source is 'reliable' and the other falls victim to 'under-reporting', whereas clearly this bias is situational.

It is also not impossible that other features of 'objective' statistical databases are produced by the situatedness of interpretation as well as of discourse: and this is particularly the case with confusing or incoherent coding matrices. The writer J.L. Borges once created a fictitious classificatory system of animals thus: 'Animals are divided into those that belong to the Emperor: embalmed ones: those that are trained: suckling pigs: mermaids: fabulous ones: stray dogs: those that are included in this classification: those that tremble as if they were mad: innumerable ones: those drawn with a very fine camel's hair brush: others: those that have just broken a flower vase: those that resemble flies from a distance' (Borges, 1964, 108). This is clearly absurd, but many 'coding' matrices used in safety management are not much better, the key problem being most events could fit plausibly into any category. This will again confuse and produce incoherent data. Lakoff (1990) a proponent of situated embodied cognition argues that incoherent categories arise from failure to consider this view of cognition. In our own research we would stress that reliable taxonomies are best produced from coherent approaches to cognition and activity, and that the situated, sociated view of cognition seems to provide such a view. 


\section{Bibliography}

Atkinson, R.C., and Shiffrin, R.M. 1971, The Control of Short Term Memory Scientific American, 225, 2, 82-92

Baddeley, A. 1986, Working Memory, (Oxford University Press, Oxford)

Barba, G. 1999. Memory, consciousness and temporality: what is retrieved and who exactly is controlling the retrieval? In E. Tulving (ed.) Memory, Consciousness and the Brain. (Psychology Press, London).

Bechtel, W., and Abrahamsen, A. 2002, Connectionism and the Mind (Blackwell, Oxford)

Bekerian, D.A., and Baddeley, A.D. 1980. Saturation advertising and the repetition effect. Journal of Verbal Learning and Verbal Behaviour, 19, 17-25.

Borges J.L. 1964, Other Inquisitions (University of Texas Press, Austin)

Clancey, W., 1997. Situated Cognition, (CUP, Cambridge)

Craik, F.I.M. \& Lockhart, R.S. (1972) Levels of processing: A framework for memory research, Journal of Verbal Learning and Verbal Behavior, 11, 671684

Cummings, T.G., \& Worley, C.G. (1997) Organization development and change, (South Western, Chicago)

Davies, J. B. 1992, The Myth of Addiction, (Harwood Academic Publishers, London)

Davies, J.B. 1997, Drugspeak (Martin Dunitz, London).

Davies, J.B., Ross, A., Wallace, B., Wright, L. in press. Safety Management: A Qualitative Safety Approach (Taylor and Francis, London)

Dreyfus, H. 1992 What Computers Still Can't Do, (MIT Press, NY)

Glenberg, A.M. 1997. What memory is for, Behavioral and Brain Sciences 20, 1, 155. Online http://www.bbsonline.org/documents/a/00/00/05/54/bbs0000055400/bbs.glenberg.html

Hayes, N. 2000, Foundations of Psychology, (Thomson, London)

Hollnagel, E. Cognition as control: A pragmatic approach to the modelling of joint cognitive systems. IEEE Journal of Systems, Man and Cybernetics (in press). Online: http://www.ida.liu.se/ eriho/Publications_O.htm

Lakoff, G. 1990, Women, Fire and Dangerous Things, (UCP, Chicago)

Lakoff, G., and Johnson, M. 1999, Philosophy in the Flesh. (Basic Books, New York)

Loftus, G.R. and Loftus, E.F. 1975, Human Memory: The Processing of Information. (Halsted Press New York)

Morris, P.E. 1982, Research on Memory in Everyday Life. In P. Sanders, N. Hayes, R. Brody, and L. Jones (eds) A Handbook for GCSE Psychology Students (ATP Publications, Leicester)

Reisberg, D., Rappaport, I. \& O'Shaugnessy, M. 1984, Limits of working memory: the digit digit-span. Journal of Experimental Psychology: Learning, Memory, and Cognition, 10, 203-221

Shallice T. and Warrington, E.K. 1970, Independent functioning of verbal memory stores: a neurophysiological study. Quarterly Journal of Experimental Psychology, 22, 261, 273

Sougné, J.P. (Submitted). Short Term Memory in a Network of Spiking Neurons. Neural Computation and Psychology Worshop NCPW7 Brighton, Online http://www.ulg.ac.be/cogsci/jsougne/ncpw7.pdf

Wallace, B., Ross, A., Davies, J.B., Wright, L. 2002, Information, Arousal and Control in the UK Railway Industry. In A. Thatcher, J. Fisher, K. Miller (eds) Proceedings of CybErg 2002: The Third International Cyberspace Conference on Ergonomics. (IAE Press, Johannesburg)

Wilcox S., and Katz S.(1981) A direct realist alternative to the traditional conception of memory. Behaviorism 9: 227-239

Wright, L., Ross, A., Davies, J.B. 2001, SPAD Risk Factors: Results of a Focus Group Study. 
(Railway Safety, London). Available on 'Human Factors Research Catalogue CDROM, July 2001'

Zadny, J., and Gerard, H.B. 1974. Attributed Intentions and Informational Selectivity, Journal of Experimental Social Psychology, 10, 34-52 